\title{
Small Intestinal Anastomotic Leakage
}

National Cancer Institute

\section{Source}

National Cancer Institute. Small Intestinal Anastomotic Leakage. NCI Thesaurus. Code C78612.

Leakage due to breakdown of a small intestinal anastomosis. 